\title{
The Economic Consequences of Financial Institution Structural Changes
}

\author{
Young-Jin Kim
}

\section{Introduction}

2. The Model

3. The Movements of the Four Interest Rates

4. Relaxation of a Constant Level of Deposit Assumption

5. Wealth Implication of NOW Accounts

6. Conclusion

\section{INTRODUCTION}

There have been a lot of discussions about a more competitive and efficient financial system through the removal of regulations governing the financial institutions. The regulations on financial institutions have been justified on the ground that those regulations will enhance safety of depositors and prevent an excessive competition among financial institutions. However, it is generally agreed that the lack of competition, inefficiency, disintermediation, and deteriorating performance of institutions are the results from the unwarranted regulations on financial institutions. Several government studies also recommended unanimously the removal of all these regulations which impair the free competition among the institutions as the only solution for the efficiency and survival of financial system. Recent movements along this line of thoughts include the possible national adoption of NOW accounts, introduction of longterm mortgage loans by credit unions and the gradual removal of regulation $Q$, just to name a few.

These expected structural changes in financial institutions have raised a

Author: Assistant Professor, School of Management, Seoul National University 
lot of concern of scholars and managers of these institutions. Since these changes will affect the amount of funds available for different types of loans offered by different types of institutions, their impacts on real capital investment mix as well as on financial institutions could be substantial. Although there have been some effort to evaluate the effects of these changes on the performance and behavior of financial institutions involved, little emphasis has been placed on the macroeonomic impact of these changes on the real capital investment mix and the resulting wealth implication due to the lack of understanding the relationship between real and financial sector.

Traditionally, the explanation about the relationship between the financial and real sector has been based on ad hoc discussions or partial equilibrium analytics. The classical and Keynesian approaches have regarded the quantity of money or long-term bond rate as the only linkage between the financial sector and the real sector and expressed concern over the impact of this kind of change only on quantity of money and long-term bond rate. However, it is difficult and inappropriate to determine the overall impact of these changes with a theory which does not give explicit recognition to financial intermediaries. This inadequacy reflects a common tendency of classical and Keynesian economics to place a secondary importance on the role of financial sector.

Contrary to the traditional approach, Gurley and Shaw (1960) claim that the essential features of the relationship between the financial system and the real economy are not the long-term interest rate or the quantity of money, but the demand and supply relationships of the whole spectrum of assets and the structure of interest rates and asset yields. Along this line, Tobin and Brainard (1963) apply a financial sector general equilibrium analysis incorporating the behavioral assumptions of each sector in the economy. They pointed out that the drawbacks of the partial equilibrium model are that it ignores: (1) the portfolio adjustment process of each sector in the economy, (2) the interdependence of various markets and intermediaries, and (3) the rational behavior for portfolio choices of individuals and the financial sector. Tobin and Brainard 
(1962) referred to the rate of return at which individuals hold the existing stock of capital as the required rate of return and concluded that it is this required rate of return which relates the financial sector and real economic activity. Tobin (1969) refined his financial sector general equilibrium model by noting that the level of investment activities are directly related to the required rates of return on assets. This method of relating the financial sector and real economic activity through the required rates of return on assets is utilized by Rao and Kaminow (1973) to analyze the impact of asset reserve requirements on required rate of returns on housing and real and their implication on the real investment mix. They concluded that the necessary condition for the changes in required rates of return to have any impact on real investment is the low degree of substitutions among holdings of different types of real assets.

The analysis taken in this paper will similarly employ a general equilibrium model with a financial sector, and explicitly recognizes the function of demand and supply relationship in the financial sector. In this analysis, a specific example of the introduction of NOW accounts is chosen to evaluate its impacts on real capital investment mix and its wealth implication. However, any structural change can be applied to this model without losing any generality of the model. Section 2 describes the model adopted in the analysis. Section 3 looks at the effects of NOW accounts as a structural change on the movements of interest rates and the level of real investments. Section 4 evaluates the significance of the assumption of constant deposit level on the interest rates and on the level of real investments by relaxing the assumption. Section 5 looks at the wealth implication of the resulting changes in the real investment mix. Section 6 concludes the paper by summarizing the results.

\section{THE MODEL}

This section describes the general equilibrium model with financial sector 
which is employed in this study.

\section{1) Assets and Liabilities of Each Sector}

The balance sheets of Table 1 define the nature of the economy assumed for this study. This economy contains three sectors: govetnment, financial intermediaries, and the remaining sector which includes households and firms. There will be six assets in the analysis: reserves, deposits, two real assets (houses and other real capital), and two financial assets(mortgages and loans).

The government sector, which includes a monetary authority, is assumed to have only one liability, reserves. In this sense, the government can be viewed as a debtor in the economy. The financial intermediaries sector places its resources in mortgage and other industrial loans besides the reserves. It holds deposits from the other sector as a liability. The stock of reserves is assumed to be exogenously determined by the monetary authority, and intermediaries passively accept whatever quantity is made available. Thus, the reserves market is always in equilibrium. The remaining sector of households and firms holds deposits, houses, and real capital as assets, and mortgage loans and other industrial loans as liabilities. For simplicity, it will be assumed that there is only one kind of each asset and liability included in the analysis. This assumption of homogeneity of assets and liabilities will not impair the qualitative conclusions of this analysis.

\section{2) Rates}

The balance sheets of Table 1 contain a total of six assets; reserves, deposits, houses, capital, mortgages, and other loans. The yield on these assets are denoted respectively as $r_{c}, i_{d}, r_{h}, r_{k}, i_{m}$, and $i_{b}$. However, the yield on reserves is zero and the yield on deposits is assumed to be institutionally fixed.

Table 1. Sectoral Balance Sheets

Government

Reserve

Financial Intermediaries

\begin{tabular}{l|l} 
Reserve & Deposits \\
Mortgages & \\
Loans &
\end{tabular}

Other Sector (Households and firms)

\begin{tabular}{l|l}
\hline Deposits & Mortgages \\
Houses & Loans \\
Capital & Net Worth
\end{tabular}


Hence, only four rates, $i_{m}, i_{b}, r_{k}, r_{k}$ will be determined in the market by the supply and demand relationship of their associated assets. Further, it will be assumed throughout the analysis that the general level of prices is fixed.

\section{3) Notation}

\section{Sectors}

$G$ : Government.

$F:$ Financial intermediaries.

$E:$ The rest of the economy other than government and financial intermediaries. Households and the firms are included.

Assets

$R:$ Reserves. The supply of reserves is fixed in this analysis.

$D$ : Deposits. For simplicity, only one type of deposit is assumed.

$M$ : Mortgage loans supplied by financial intermediaries.

$B$ : Capital loans supplied by financial intermediaries.

$H$ : Houses (measured in terms of current market value).

$K:$ Real capital, except houses.

$W:$ Equity of households.

Rates

$i_{m}$ : Rate of interest financial intermediaries charge on mortgage loans.

$i_{b}$ : Rate of interest financial intermediaries charge on capital loans.

$r_{h}$ : Required rate of return on housing

$r_{k}$ : Required rate of return on real capital except housing.

$i_{d}$ : Rate of interest on deposits which is exogenously fixed by regulation.

Shift Parameter

$\theta:$ a specific structural change (in this analysis, NOW account introduction)

\section{4) Equations}

To satisfy the equilibrium condition that the demand for an asset is equal to supply of the asset, the market clearing conditions for the assets and liabilities of the model are the following. For convenience, the signs of partial derivatives of each function with respect to each of its arguments appear above 
the rates.

Deposit Market:

$$
D_{f}^{s}\left(i_{m}^{+}, i_{b}^{+}, i_{d}^{-}, R^{+}\right)=D_{e}^{d}\left(i_{m}^{--}, i_{b}^{-}, r_{h}^{-}, r_{k}^{-}, i_{d}^{+}\right)
$$

Mortgage Market:

$$
M_{e}^{s}\left(i_{m}{ }^{-}, i_{b}{ }^{+}, r_{h}{ }^{+}, r_{k}{ }^{+}, i_{d}{ }^{+}\right)=M_{f^{d}}\left(i_{m}{ }^{+}, i_{b}^{-}, R^{+}, \theta^{+}, i_{d}^{-}\right)
$$

Capital Loan Market:

$$
B_{e}^{s}\left(i_{m}{ }^{+}, i_{b}^{-}, r_{h}{ }^{+}, r_{k}^{+}, i_{d}{ }^{+}\right)=B_{f^{d}}\left(i_{m}{ }^{-}, i_{b}{ }^{+}, R^{+}, \theta^{-}, i_{d}^{-}\right)
$$

Housing Market:

$$
H_{s}\left(r_{h}^{-}\right)=H_{e}\left(i_{m}^{-}, i_{b}^{-}, r_{h}^{+}, r_{k}^{-}\right)
$$

Real Capital Market:

$$
K_{s}\left(r_{k}^{-}\right)=K_{e}\left(i_{m}{ }^{-}, i_{b}, r_{h}^{-}, r_{k}^{+}\right)
$$

The subscripts, $f$ and $e$, denote the financial intermediary sector and the household-firm sector respectively. The superscripts, $d$ and $s$, mean demand and supply for the sector in question.

The four rates, $i_{m}, i_{b}, r_{h}$ and $r_{k}$, which will be determined simultaneously, will influence the portfolio composition chosen by the households and firm sector. The portfolio composition of the financial intermediaries is influenced by changes in $i_{m}$ and $i_{b} . \theta$, the shift parameter, was included in the equations as an argument of $M_{f}^{d}$ and $B_{f}^{d}$. Since the introduction of NOW accounts will cause transfer of funds from commercial banks to thrift institutions due to commercial banks' loss of the monopoly power over the funds held for transaction purposes, and since thrift institutions hold mortgage loans as their primary asset, the introduction of NOW accounts will change the demand characteristics of financial intermediaries sector to more demand for mortgage loans and less demand for other capital loans.

$H\left(r_{h}\right)$ and $K\left(r_{k}\right)$ are used to show that the level of housing and capital are dependent on the market rates of return on houses, $r_{h}$, and capital, $r_{k}$. The signs of the partial derivatives are specified by the assumption that (a) all assets are gross substitutes and (b) each real asset is financed by its associated financial asset. The partial derivative of each equation with respect to 
its own rate of interest is positive for demand and negative for supply relationships; the opposite holds for derives with respect to cross rates. Intermediary demand for mortgage loans is positively related to the shift parameter, $\theta$, and the opposite holds for capital loans.

\section{5) Behavioral Assumptions of Demand Functions}

The principle assumption about the demand behavior of each sector in this study is that all assets are gross substitutes such that:

$$
\begin{aligned}
& \text { a. } \frac{\partial D_{i}}{\partial R_{i}}>0, \quad \frac{\partial D_{i}}{\partial R_{j}} \leq 0, \quad i \neq j \\
& \text { b. } \frac{\partial D_{i}}{\partial R_{i}^{-}}=\sum \underset{\substack{j=1, \ldots, n \\
i \neq j}}{=} \partial D_{j}
\end{aligned}
$$

where $R_{i}$ and $D_{i}$ denote return on asset $i$ and demand for asset $i$ respectively. The gross substitutes assumption and the balance sheet identity necessitate the assumption that increases in demand for one asset will be completely offset by decreases in demand for other assets for each sector. These two assumptions are necessary to infer the signs of the partial derivatives in the manipulation of market-clearing conditions.

\section{6) Balance Sheet Identity}

The financial intermediary sector and the househcds and firm sector must always meet the balance sheet constraint:

Asset $=$ Liabilities + Net Worth

For the financial intermediary sector,

$$
M_{f}+B_{f}+R=D_{f}+R
$$

For the households and firm sector,

$$
D_{e}+H_{e}+K_{e}=M_{e}+B_{e}+W
$$

where net worth is defined to be:

$$
W=H\left(r_{h}\right)+K\left(r_{k}\right)
$$

\section{THE MOVEMENTS OF THE FOUR INTEREST RATES}

With the model described above, the main concern of this analysis is to 
show the movements of the four interest rates, $i_{m}, i_{b}, r_{h}$, and $r_{k}$, which will change the level of the associated financial and real assets. As was explained earlier, the above four interest rates will be simultaneously determined in the market in such a way that the demand for and supply of each asset will be matched. It is of particular interest to determine whether a structural change of the adoption of NOW accounts implies an increased level of housing and decreased level of other real capital.

Following the notion that the level of investment in housing and other real capital in this analysis on the required rate of return on those assets, increases in these real assets will be possible only if NOW accounts lower the rate of return for these assets. These conditions can be written as:

$$
\frac{d R_{i}}{d \theta}<0, \quad i=k, h
$$

These are necessary and sufficient conditions for houses and other real capital to increase. The changes in the level of financial assets can also be found by looking at the derivatives $\frac{d i_{m}}{d \theta}$, and $\frac{d i_{b}}{d \theta}$, for mortgage and other loans respectively.

In order to find the signs of the four derivatives, $\frac{d i_{m}}{d \theta}, \frac{d i_{b}}{d \theta}, \frac{d r_{h}}{d \theta}$, and $\frac{d r_{k}}{d \theta}$, market-clearing conditions for each asset and demand behavior assumptions will be utilized. Since there are five equations for market-clearance of assets and there are four interest rates which will be determined in the system of equation, one equation will be redundant. This can be shown from the fact that the clearrance of the market for four assets will automatically guarantee the equilibrium of the fifth asset market, which comes from the balance sheet identities of the financial intermediaries sector and the other sector. Given the concern of this analysis, the deposit market is dropped.

Totally differentiating the market clearing conditions and rearranging in matrix form leads to: 


$$
\left(\begin{array}{cccc}
\frac{\partial M_{e}-\partial M_{f}}{\partial i_{m}} & \frac{\partial M_{e}-\partial M_{f}}{\partial i_{b}} & \frac{\partial M_{e}}{\partial r_{h}} & \frac{\partial M_{e}}{\partial r_{k}} \\
\frac{\partial B_{e}-\partial B_{f}}{\partial i_{m}} & \frac{\partial B_{e}-\partial B_{f}}{\partial i_{b}} & \frac{\partial B_{e}}{\partial r_{h}} & \frac{\partial B_{e}}{\partial r_{k}} \\
-\frac{-\partial H_{e}}{\partial i_{m}} & \frac{-\partial H_{e}}{\partial i_{b}} & \frac{\partial\left(H-H_{e}\right)}{\partial r_{h}} & \frac{-\partial H_{e}}{\partial r_{k}} \\
\frac{-\partial K_{e}}{\partial i_{m}} & -\frac{-\partial K_{e}}{\partial i_{b}} & -\frac{-\partial K_{e}}{\partial r_{h}} & \frac{\partial\left(K-K_{e}\right)}{\partial r_{k}}
\end{array}\right)\left(\begin{array}{c}
d i_{m} \\
d i_{b} \\
d r_{h} \\
d r_{k}
\end{array}\right)=\left(\begin{array}{c}
\frac{\partial M_{f}}{\partial \theta} d \theta \\
\frac{\partial B_{f}}{\partial \theta} d \theta \\
0 \\
0
\end{array}\right)
$$

Given the fixed level of $R$ and $i_{d}$, the partials of the assets with respect to these changes were ignored. By dividing the above system of equations by $d \theta$, it can be rewritten as:

$$
\left[A_{i j}\right]\left(\begin{array}{c}
\frac{d i_{m}}{d \theta} \\
\frac{d i_{b}}{d \theta} \\
\frac{d r_{h}}{\partial \theta} \\
\frac{d r_{k}}{d \theta}
\end{array}\right)=\left(\begin{array}{c}
\frac{\partial M_{f}}{\partial \theta} \\
\frac{\partial B_{f}}{\partial \theta} \\
0 \\
0
\end{array}\right)
$$

where is the matrix of partials of the above expression. Thus, we can simplify the above equation as:

$$
A \cdot X=S
$$

where $X$ is the column vector of the four derivatives.

From the above expression, four derivatives, $\frac{d i_{m}}{\partial \theta}, \frac{d i_{b}}{d \theta}, \frac{d r_{h}}{d \theta}$, and $\frac{d r_{k}}{d \theta}$ can be found by using Cramer's rule:

$$
x_{i}=\frac{\left|A_{i}\right|}{|A|}
$$

where $A_{i}$ is the matrix formed by replacing the column of the $A$ matrix with the vector $S$.

Since the concern of the analysis involves the signs of the derivatives, not their magnitude, it is necessary only to determine the signs of the numerator $\left|A_{i}\right|$ and denominator $|\mathrm{A}|$ to obtain the signs of the changes of level of financial and real assets in the system. It can be shown that the sign of the determinant of matrix $A$ is dependent on the order of the determinant since matrix $A$ is a gross substitution matrix ${ }^{2}$, or more broadly, the Metzler 
matrix, which was shown by Metzler(1945). Therefore, the sign of the determinant of matrix $A$ is pcsitive. This implies that the sign of $\left|A_{i}\right|$ will be same as those of $X_{i s}$.

The column vector, $S$, which will replace the $i^{\text {in }}$ column of matrix $A$, has special characteristics in its elements. Since $M_{f}$ and $B_{f}$ are competitive assets for the portfolio of the financial intermediaries sector, an increase in $M_{f}$ will be made at the expense of a decrease in $B_{f}$, if there is no change in the level of deposits. By assuming the same reserve requirements, no change in hoarding and velocity, and the homogeneity of deposits in the system, the transfer of deposits from commercial banks to thrift institutions by the introduction of NOW accounts will not change the level of deposits in the economy. The above assumptions lead to the following equation:

$$
\frac{\partial M_{f}}{\partial \theta}+\frac{\partial B_{f}}{\partial \theta}=0
$$

This assumption implies that the increase in demand for mortgages by financial intermediaries will be completely offset by the decrease in demand for other loans by financial intermediaries.

By using the characteristics of balance sheet identity for each sector, expression $A \cdot X=S$ can be changed as follows ${ }^{3}$ :

$$
\left(\begin{array}{cccc}
\frac{\partial M_{e}-\partial M_{f}}{\partial i_{m}} & \frac{\partial M_{e}-\partial M_{f}}{\partial i_{b}} & \frac{\partial M_{e}}{\partial r_{h}} & \frac{\partial M_{e}}{\partial r_{k}} \\
\frac{\partial D_{e}-\partial D_{f}}{\partial i_{m}} & \frac{\partial D_{e}-\partial D_{f}}{\partial i_{b}} & \frac{\partial D_{e}}{\partial r_{h}} & \frac{\partial D_{e}}{\partial r_{k}} \\
\frac{-\partial H_{e}}{\partial i_{m}} & \frac{-\partial H_{e}}{\partial i_{b}} & \frac{\partial\left(H-H_{e}\right)}{\partial r_{h}} & \frac{-\partial H_{e}}{\partial r_{k}} \\
\frac{-\partial K_{e}}{\partial i_{m}} & -\frac{\partial K_{e}}{\partial i_{b}} & \frac{-\partial K_{e}}{\partial r_{h}} & \frac{\partial\left(K-K_{e}\right)}{\partial r_{k}}
\end{array}\right)\left(\begin{array}{c}
\frac{d i_{m}}{d \theta} \\
\frac{d i_{b}}{d \theta} \\
\frac{d r_{h}}{d \theta} \\
\frac{d r_{k}}{d \theta}
\end{array}\right)=\left(\begin{array}{c}
\frac{\partial M_{f}}{\partial \theta} \\
0 \\
0 \\
0
\end{array}\right)
$$

Cramer's rule may now be used for determining the signs for $i=1,2,3,4$; $\frac{d i_{m}}{d \theta},-\frac{d i_{b}}{d \theta}, \frac{d r_{h}}{d \theta}, \frac{d r_{k}}{d \theta}$ respectively.

The signs of $|A i|$ for $i=1,2,3,4$ will be calculated as following:

$$
\left|A_{1}\right|=(-1)^{2} s_{1} \mid \begin{array}{lcc}
\frac{\partial D_{e}-\partial D_{f}}{\partial i_{b}} & \frac{\partial D_{e}}{\partial r_{h}} & \frac{\partial D_{e}}{\partial r_{k}} \\
\frac{-\partial H_{e}}{\partial i_{b}} & \frac{\partial\left(H-H_{e}\right)}{\partial r_{h}} & \frac{-\partial H_{e}}{\partial r_{k}}
\end{array}
$$




$$
\begin{gathered}
\left|\frac{-\partial K_{e}}{\partial i_{b}} \frac{-\partial K_{e}}{\partial r_{k}} \frac{\partial\left(K-K_{e}\right)}{\partial r_{k}}\right| \\
\text { where } s_{1}=-\frac{\partial M_{f}}{\partial \theta}>0 \\
=(-1)^{2} s_{1}\left[\frac{\partial D_{e}-\partial D_{f}}{\partial i_{b}}\left(\frac{\partial\left(H-H_{e}\right)}{\partial r_{h}} \cdot \frac{\partial\left(K-K_{e}\right)}{\partial r_{k}}-\frac{-\partial H_{e}}{\partial r_{k}} \cdot \frac{-\partial K_{e}}{\partial r_{h}}\right)\right. \\
+\frac{\partial D_{e}}{\partial r_{k}}\left(\frac{-\partial H_{e}}{\partial r_{k}} \cdot \frac{-\partial K_{e}}{\partial i_{b}}-\frac{-\partial H_{e}}{\partial i_{b}} \cdot \frac{\partial\left(K-K_{e}\right)}{\partial r_{k}}\right) \\
\left.+\frac{\partial D_{e}}{\partial r_{k}}\left(\frac{-\partial H_{e}}{\partial i_{b}} \cdot \frac{-\partial K_{e}}{\partial r_{k}}-\frac{\partial\left(H-H_{e}\right)}{\partial r_{h}} \cdot \frac{-\partial K_{e}}{\partial i_{b}}\right)\right]
\end{gathered}
$$

Here, it is shown that $\frac{d i_{m}}{d \theta}$ has a negative sign which means the adoption of NOW accounts will lower the interest rate on mortgage loans. The sign of $\frac{d i_{b}}{d \theta}$ can be shown in a similar fashion:

$$
\begin{aligned}
\left|A_{2}\right|=(-1)^{3} s_{1}\left|\begin{array}{ccc}
\frac{\partial D_{e}-\partial D_{f}}{\partial i_{m}} & \frac{\partial D_{e}}{\partial r_{k}} & \frac{\partial D_{e}}{\partial r_{k}} \\
\frac{-\partial H_{e}}{\partial i_{m}} & \frac{\partial\left(H-H_{e}\right)}{\partial r_{h}} & \frac{-\partial H_{e}}{\partial r_{k}} \\
\frac{-\partial K_{e}}{\partial i_{m}} & \frac{-\partial K_{e}}{\partial r_{h}} & \frac{\partial\left(K-K_{e}\right)}{\partial r_{k}}
\end{array}\right| \\
=(-1)^{3} s^{1}\left[\frac{\partial D_{e}-\partial D_{f}}{\partial i_{m}}\left(\frac{\partial\left(H-H_{e}\right)}{\partial r_{h}} \cdot \frac{\partial\left(K-K_{e}\right)}{\partial r_{k}}-\frac{-\partial H_{e}}{\partial r_{k}} \cdot \frac{-\partial K_{e}}{\partial r_{k}}\right)\right. \\
+\frac{\partial D_{e}}{\partial r_{h}}\left(\frac{-\partial H_{e}}{\partial r_{k}} \cdot-\frac{-\partial K_{e}}{\partial i_{m}}-\frac{-\partial H_{e}}{\partial i_{m}} \cdot \frac{\partial\left(K-K_{e}\right)}{\partial r_{k}}\right) \\
\left.+\frac{\partial D_{e}}{\partial r_{h}}\left(-\frac{-\partial H_{e}}{\partial i_{m}} \cdot \frac{-\partial K_{e}}{\partial r_{h}}-\frac{\partial\left(H-H_{e}\right)}{\partial r_{h}} \cdot \frac{-\partial K_{e}}{\partial i_{m}}\right)\right]
\end{aligned}
$$

The sign in the large bracket is negative, and, consequently, the sign of $\frac{d i_{b}}{d \theta}$ is positive. Thus, the interest rate on capital loans will increase due to NOW accounts.

The most important part of this analysis is the sign $\frac{d r_{h}}{d \theta}$. This sign will indicate whether NOW accounts will, in fact, increase housing. Since it is noted that the level of housing is dependent upon the required rate of return from housing, the level of $r_{h}$ will decide the level of housing in equilibrium. 
Following the same procedure for $\left|A_{1}\right|$ and $\left|A_{2}\right|$,

$$
\begin{gathered}
\left|A_{3}\right|=(-1)^{4} s_{1}\left|\begin{array}{ccc}
\frac{\partial D_{e}-\partial D_{f}}{\partial i_{m}} & \frac{\partial D_{e}-\partial D_{f}}{\partial i_{b}} & \frac{\partial D_{e}}{\partial r_{k}} \\
\frac{-\partial H_{e}}{\partial i_{m}} & \frac{-\partial H_{e}}{\partial i_{b}} & \frac{-\partial H_{e}}{\partial r_{k}} \\
\frac{-\partial K_{e}}{\partial i_{m}} & \frac{-\partial K_{e}}{\partial i_{b}} & \frac{\partial\left(K-K_{e}\right)}{\partial r_{k}}
\end{array}\right| \\
=(-1)^{4} s_{1}\left[\frac{\partial D_{e}-\partial D_{f}}{\partial i_{m}}\left(\frac{-\partial H_{e}}{\partial i_{b}} \cdot \frac{\partial\left(K-K_{e}\right)}{\partial r_{k}}-\frac{-\partial H_{e}}{\partial r_{k}} \cdot-\frac{\partial K_{e}}{\partial i_{b}}\right)\right. \\
+\frac{\partial D_{e}-\partial D_{f}}{\partial i_{b}}\left(-\frac{\partial H_{e}}{\partial r_{k}} \cdot \frac{-\partial K_{e}}{\partial i_{m}}-\frac{\partial H_{e}}{\partial i_{m}} \cdot \frac{\partial\left(K-K_{e}\right)}{\partial r_{k}}\right) \\
\left.+\frac{\partial D_{e}}{\partial r_{k}}\left(\frac{-\partial H_{e}}{\partial i_{m}} \cdot \frac{-\partial K_{e}}{\partial i_{b}}-\frac{-\partial H_{e}}{\partial i_{b}} \cdot \frac{-\partial K_{e}}{\partial i_{m}}\right)\right]
\end{gathered}
$$

The sign of $\left|A_{4}\right|$ will determine whether NOW accounts will lower the level of real capital except houses. $\left|A_{4}\right|$ is:

$$
\begin{gathered}
\left|A_{4}\right|=(-1)^{5} s_{1}\left|\begin{array}{ccc}
\frac{\partial D_{e}-\partial D_{f}}{\partial i_{m}} & \frac{\partial D_{e}-\partial D_{f}}{\partial i_{b}} & \frac{\partial D_{e}}{\partial r_{h}} \\
-\frac{\partial H_{e}}{\partial i_{m}} & \frac{-\partial H_{e}}{\partial \bar{i}_{b}} & \frac{\partial\left(H-H_{e}\right)}{\partial r_{h}} \\
-\frac{\partial K_{e}}{\partial i_{m}} & \frac{-\partial K_{e}}{\partial i_{b}} & \frac{-\partial K_{e}}{\partial r_{h}}
\end{array}\right| \\
=(-1)^{5} s_{1}\left[\frac{\partial D_{e}-\partial D_{f}}{\partial i_{m}}\left(\frac{-\partial H_{e}}{\partial i_{b}} \cdot-\frac{\partial K_{e}}{\partial r_{h}}-\frac{\partial\left(H-H_{e}\right)}{\partial r_{h}} \cdot \frac{-\partial K_{e}}{\partial i_{b}}\right)\right. \\
+\frac{\partial D_{e}-\partial D_{f}}{\partial i_{b}}\left(-\frac{-\partial K_{e}}{\partial i_{m}} \cdot \frac{\partial\left(H-H_{e}\right)}{\partial r_{h}}-\frac{-\partial H_{e}}{\partial i_{m}} \cdot \frac{-\partial K_{e}}{\partial r_{h}}\right) \\
\left.+\frac{\partial D_{e}}{\partial r_{h}}\left(-\frac{\partial H_{e}}{\partial i_{m}} \cdot-\frac{-\partial K_{e}}{\partial i_{b}}-\frac{-\partial H_{e}}{\partial i_{b}} \cdot \frac{-\partial K_{e}}{\partial i_{m}}\right)\right]
\end{gathered}
$$

In order to decide the signs of expressions $\left|A_{3}\right|$ and $\left|A_{4}\right|$ without resorting to the extreme cases of specialization and dichotomy, ${ }^{4}$ we have to use the "adjacency property" which was supported by Modigliani, Rasche and Cooper (1970), Feige and Parkin (1971) and Santomero (1974). They argue that non-adjacent assets are insensitive to one another compared with adjacent assets. The adjacency property leads to the following inequalities: 


$$
\frac{-\partial H_{e}}{\partial i_{m}}>\frac{-\partial H_{c}}{\partial i_{b}}, \frac{-\partial K_{e}}{\partial i_{b}}>\frac{-\partial K_{e}}{\partial i_{m}} \longrightarrow \text { low substitutability }
$$

which means demand for houses and real capital will be much more affected by $i_{m}$ and $i_{b}$ than by $i_{b}$ and $i_{m}$, respectively. These inequalities make the sign of ambiguous parts in the expressions for $\left|A_{3}\right|$ and $\left|A_{4}\right|$ positive. For $\left|A_{3}\right|$, the comparison between the first and second term in absolute value can be obtained by comparing the magnitude between $\frac{-\partial H_{e}}{\partial i_{b}}$ and $\frac{-\partial H_{e}}{\partial i_{m}}$ and between $\frac{-\partial K_{e}}{\partial i_{b}}$ and $\frac{-\partial K_{e}}{\partial i_{m}}$. Since the absolute value of $\frac{\partial\left(K-K_{e}\right)}{\partial r_{k}}$ is greater than that of $\frac{-\partial H_{e}}{\partial r_{k}}$, the size of $\frac{-\partial H_{e}}{\partial i_{b}}$ and the size of $\frac{-\partial H_{e}}{\partial i_{m}}$ play the most important role in deciding the sign of $\left|A_{3}\right|$, even though the sizes of $\frac{-\partial K_{c}}{\partial i_{b}}$ and $\frac{-\partial K_{e}}{\partial i_{m}}$ play a part. Since $\frac{-\partial H_{e}}{\partial i_{m}}>\frac{-\partial H_{e}}{\partial i_{b}}$ is posited, the claim may be made that the absolute value of the second term is greater than of the first so that the sign of $\left|A_{3}\right|$ is negative.

The sign of $\left|A_{4}\right|$ can be approached in the same way. Just as was the case with $\left|A_{3}\right|$, the third term will be negative. Considering the absolute value of $\frac{-\partial K_{e}}{\partial i_{b}}$ and $\frac{-\partial K_{e}}{\partial i_{m}}$, it will be noted that the absolute value of the first term is greater than that of the second term. Therefore, the sign of $\left|A_{4}\right|$ is positive.

\section{RELAXATION OF A CONSTANT LEVEL OF DEPOSIT ASSUMPTION}

The assumption made earlier about the level of deposits when NOW accounts are introduced will be examined further in this section. The possible transfer of funds from demand deposits to savings deposits indicates that the funds transferred are now subject to lower reserve requirements than before, because the reserve requirement on savings deposits is lower than of demand deposits. Thus, funds subject to a low reserve requirement will increase deposits over 
the previous level of deposits. This can be written as follows:

$$
\frac{\partial M_{f}}{\partial \theta}+\frac{\partial B_{f}}{\partial \theta}>0
$$

which allows the increase in the size of the balance sheet of the financial intermediaries' sector.

The question now to be addressed is how this increase in deposits will affect the interest rates considered in the analysis and the level of financial and real assets. The relaxation of the assumption of no increase in the level of deposits adds an additional expression to the calculation of the signs of the four derivatives.

From the previous expressions for $\left|A_{1}\right|,\left|A_{2}\right|,\left|A_{3}\right|$, and $\left|A_{4}\right|$, the following terms should be added respectively.

For $\left|A_{1}\right|$,

$$
\begin{aligned}
(-1)^{3} k & {\left[\frac{\partial M_{e}-\partial M_{f}}{\partial i_{b}}\left(\frac{\partial\left(H-H_{e}\right)}{\partial r_{k}} \cdot \frac{\partial\left(K-K_{e}\right)}{\partial r_{k}}-\frac{-\partial H_{e}}{\partial r_{k}} \cdot \frac{-\partial K_{e}}{\partial r_{k}}\right)\right.} \\
& +\frac{\partial M_{e}^{\ominus}}{\partial r_{k}}\left(\frac{-\partial H_{c}}{\partial r_{k}} \cdot \frac{-\partial K_{e}}{\partial i_{b}}-\frac{-\partial H_{e}}{\partial i_{b}} \cdot \frac{\partial\left(K-K_{e}\right)}{\partial r_{k}}\right) \\
& \left.+\frac{\partial M_{e}}{\partial r_{k}}\left(-\frac{\partial H_{e}}{\partial i_{b}} \cdot \frac{-\partial K_{e}}{\partial r_{h}}-\frac{\partial\left(H-H_{e}\right)}{\partial r_{k}} \cdot \frac{-\partial K_{e}}{\partial i_{b}}\right)\right]
\end{aligned}
$$

For $\left|A_{2}\right|$,

$$
\begin{gathered}
(-1)^{4} k\left[\frac{\partial M_{e}-\partial M_{f}}{\partial i_{m}}-\left(\frac{\partial\left(H-H_{e}\right)}{\partial r_{h}} \cdot \frac{\partial\left(K-K_{e}\right)}{\partial r_{k}}-\frac{-\partial H_{e}}{\partial r_{k}} \cdot \frac{-\partial K_{e}}{\partial r_{h}}\right)\right. \\
\quad+-\frac{\partial M_{e}}{\partial r_{h}}\left(\frac{-\partial H_{e}}{\partial r_{k}} \cdot \frac{-\partial K_{e}}{\partial i_{m}}-\frac{\oplus \partial H_{e}}{\partial i_{m}} \cdot \frac{\partial\left(K-K_{e}\right)}{\partial r_{k}}\right) \\
\left.\quad+\frac{\partial M_{e}}{\partial r_{k}}\left(\frac{-\partial H_{e}}{\partial i_{m}} \cdot \frac{-\partial K_{e}}{\partial r_{k}}-\frac{\partial\left(H-H_{e}\right)}{\partial r_{h}} \cdot \frac{-\partial K_{e}}{\partial i_{m}}\right)\right]
\end{gathered}
$$

For $\left|A_{3}\right|$,

$$
\begin{array}{r}
(-1)^{5} k\left[\frac{\partial M_{e}-\frac{\partial M_{f}}{\partial i_{m}}\left(\frac{-\partial H_{e}}{\partial i_{b}} \cdot \frac{\partial\left(K-K_{e}\right)}{\partial r_{k}}-\frac{\partial H_{e}}{\partial r_{k}} \cdot \frac{-\partial K_{e}}{\partial i_{b}}\right)}{+\frac{\partial M_{e}-\frac{\partial M_{f}}{\partial i_{b}}}{2}\left(\frac{-\partial H_{e}}{\partial r_{k}} \cdot \frac{-\partial K_{e}}{\partial i_{m}}-\frac{-\partial H_{e}}{\partial i_{m}} \cdot \frac{\partial\left(K-K_{e}\right)}{\partial r_{k}}\right)}\right.
\end{array}
$$




$$
\left.+\frac{\partial M_{e}}{\partial r_{k}}\left(\frac{-\partial H_{e}}{\partial i_{m}} \cdot \frac{-\partial K_{e}}{\partial i_{b}}-\frac{-\partial H_{e}}{\partial i_{b}} \cdot \frac{-\partial K_{e}}{\partial i_{m}}\right)\right]
$$

and for $\left|A_{4}\right|$,

$$
\begin{aligned}
(-1)^{6} k & {\left[\frac{\partial M_{e}-\partial M_{f}}{\partial i_{m}}\left(\frac{-\partial H_{e}}{\partial i_{b}} \cdot-\frac{\partial K_{e}}{\partial r_{h}}\right)-\frac{\partial\left(H-H_{e}\right)}{\partial r_{h}} \cdot-\frac{\partial K_{e}}{\partial i_{b}}\right) } \\
& +\frac{\partial M_{e}^{\oplus}-\partial M_{f}}{\partial i_{b}}\left(\frac{\partial\left(H-H_{e}\right)}{\partial r_{h}} \cdot-\frac{\partial K_{e}}{\partial i_{m}}-\frac{-\partial H_{e}}{\partial i_{m}} \cdot \frac{-\partial K_{e}}{\partial r_{h}}\right) \\
& \left.+\frac{\partial M_{e}}{\partial r_{h}}\left(-\frac{\partial H_{e}}{\partial i_{m}} \cdot \frac{-\partial K_{e}}{\partial i_{b}}-\frac{\partial H_{e}}{\partial i_{b}} \cdot \frac{-\partial K_{e}}{\partial i_{m}}\right)\right]
\end{aligned}
$$

where $k$ is the difference in value between $\frac{\partial M_{f}}{\partial \theta}$ and $\frac{\partial B_{f}}{\partial \theta}$, which is positive when we assume an increase in the level of deposits.

The signs of the additional terms incorporating the increase in deposit level imply that it-will lower the level of the four interest rates. The additional terms for $\left|A_{1}\right|$ and $\left|A_{3}\right|$ are clearly negative. Attention must be paid in deciding the signs for $\left|A_{2}\right|$ and $\left|A_{4}\right|$. In the additional expressions for $\left|A_{2}\right|$ and $\left|A_{4}\right|$, it can be shown that the diagonal elements guarantee the negative values for the terms in the brackets. In the both expressions, the first terms in the bracket dominate the other two expressions in value. Therefore, the overall signs of the additional terms for $\left|A_{2}\right|$ and $\left|A_{4}\right|$ are negative. The negative values for the additional terms indicate that an increase in the level of deposits from the introduction of NOW accounts will lower the general level of interest rates in the system.

\section{WEALTH IMPLICATION OF 'NOW' ACCOUNTS}

In the previous sections, the impact of the adoption of NOW accounts on the level of financial and real assets in the economy was analyzed by utilizing a financial sector general equilibrium model. The movements of four interest rates, $i_{m}, i_{b}, r_{k}$, and $r_{k}$ were found through comparative static analysis. The expected results from the adoption of NOW accounts are: (1) it will increase 
the availability of mortgage loans and housing; (2) it will lower the availability of industrial loans and real capital although the relaxation of the assumption of a constant level of deposits will reduce the magnitude of decrease in the availability of the two assets.

In this section, the impact of NOW accounts on the level of wealth in the economy will be ascertained. The expected changes in the level of real capital and houses, and the changes in the availability of loans for these assets imply a change in the level societal wealth, when we define the wealth as the sum of the present value of the future income streams from holding houses and real capital, plus the current level of deposits. The wealth, $W$, can be represented as following:

$$
W=\frac{r_{k}}{i_{b}} K+\frac{r_{h}}{i_{m}} H+D
$$

where $i_{b}$ and $i_{m}$ are regarded as the capitalization rates for the income stream from owning real capital and houses, respectively. $r_{k}$ and $r_{h}$ are the rates of return on real capital and houses. $D$, the level of deposits held by households and firms, is included in the above equation because it is believed to affect the societal wealth through its impact on earning power of banking industry.

In order to know how NOW accounts will change the level of wealth when it is adopted nationally, the derivative of the above wealth equation with respect to NOW accounts impact, $\theta$, was taken. By checking the components of the derivative, the change in the level of wealth can be found to be:

$$
\begin{aligned}
\frac{\partial W}{\partial \theta} & =K\left(\frac{1}{i_{b}} \cdot \frac{\partial r_{k}}{\partial \theta}-\frac{r_{k}}{i_{b}{ }^{2}} \cdot \frac{\partial i_{b}}{\partial \theta}\right)+H\left(\frac{1}{i_{m}} \cdot \frac{\partial r_{h}}{\partial \theta}-\frac{r_{h}}{i_{m}{ }^{2}} \cdot \frac{\partial i_{m}}{\partial \theta}\right) \\
& +\frac{r_{k}}{i_{b}} \cdot \frac{\partial K}{\partial \theta}+\frac{r_{h}}{i_{m}} \cdot \frac{\partial H}{\partial \theta}+\frac{\partial D}{\partial \theta}
\end{aligned}
$$

The first and second terms of the above equation explain the change in wealth caused by changes in the present value of the initial level of real capital and houses. The third and fourth terms explain the change in wealth caused by both increase and decrease in the houses and real capital, respectively. The fifth term shows the possible change in the level of deposits. The 
relaxation of the assumption of the constant deposit level will make the sign of $\frac{d D}{d \theta}$ positive.

Since the first two expressions in the brackets are changes in the net present value of real capital and houses, i.e., changes in the price of real capital and houses; and since it is known from the first section that the level of houses will increase and the level of real capital will decrease from the adoption of NOW accounts, one can conclude that the prices of real capital and houses changed in such a way that the relative price of houses is cheaper than before.

Since the first and second expressions of the derivative indicates changes in the wealth level when we hold the same combination of real capital and houses, the conclusion that the value of the first and second term will be zero can be made in the following way. Figure 1 shows that as long as the same combination of $H_{1}$ and $K_{1}$ is held, the change in the relative price, i.e., the change in the slope of the budget line, does not change the level of wealth. From this, the first two expressions can be said to equal zero.

The third and fourth expression of the derivative can tell what happened to the wealth level when there has been a shift from real capital to houses caused by a change in the relative prices. Before analyzing this, a basis, a basic assumption must be made about the relationship between the interest rates. Initially. the return on housing, $\frac{r_{h}}{i_{m}}$, is higher than that on real capital because of impediments in the supply of mortgage loans. The limited power in the asset composition for thrift institutions, an unattractive characteristics of mortgage loan such as long maturity, and relatively weak position of thrift institutions in competition for loanable funds due to the regulation $Q$ give rise to the existence of mortgage loans. Therefore, the following equation says that for portfolio equilibrium, the following holds:

$$
\frac{r_{h}}{i_{m}}-\phi=\frac{r_{k}}{i_{b}}
$$

where $\phi \geqslant 0$ was regarded as excess return form housing caused by a limited 
supply of mortgages. If NOW accounts can solve the problem of impediments in the supply of mortgage loans, $\stackrel{r_{h}}{i_{m}}=r_{i_{b}}^{r_{k}}$ will hold, which is the equilibrium condition for real and housing markets.

If this is substituted into the third and fourth expressions, the result is:

$$
\begin{aligned}
& \frac{r_{k}}{i_{b}} \cdot \frac{\partial K}{\partial \theta}+\frac{r_{h}}{i_{m}} \cdot \frac{\partial H}{\partial \theta}=\frac{r_{k}}{i_{b}} \cdot \frac{\partial K}{\partial \theta}+\left(\frac{r_{k}}{i_{b}}+\phi\right) \frac{\partial H}{\partial \theta} \\
& =-\frac{r_{k}}{i_{b}}\left(\frac{\partial K}{\partial \theta}+\frac{\partial H}{\partial \theta}\right)+\phi \cdot \frac{\partial H}{\partial \theta}
\end{aligned}
$$

since we know that $-\frac{\partial K}{\partial \theta}+\frac{\partial H}{\partial \theta}=0$ from the balance sheet identity, and $\phi \cdot \frac{\partial H}{\partial \theta}$ $>0$. This positive change in the wealth level can be drawn on the Figure 1 as a prallel line to $H^{\prime \prime} K^{\prime \prime}$, which is $H^{\prime \prime \prime} K^{\prime \prime \prime}$. This shift of budget lines indicates the increase in wealth from the third and fourth expression of the

Fig.1. The Level of Houses and Other Real Capital in the Economy

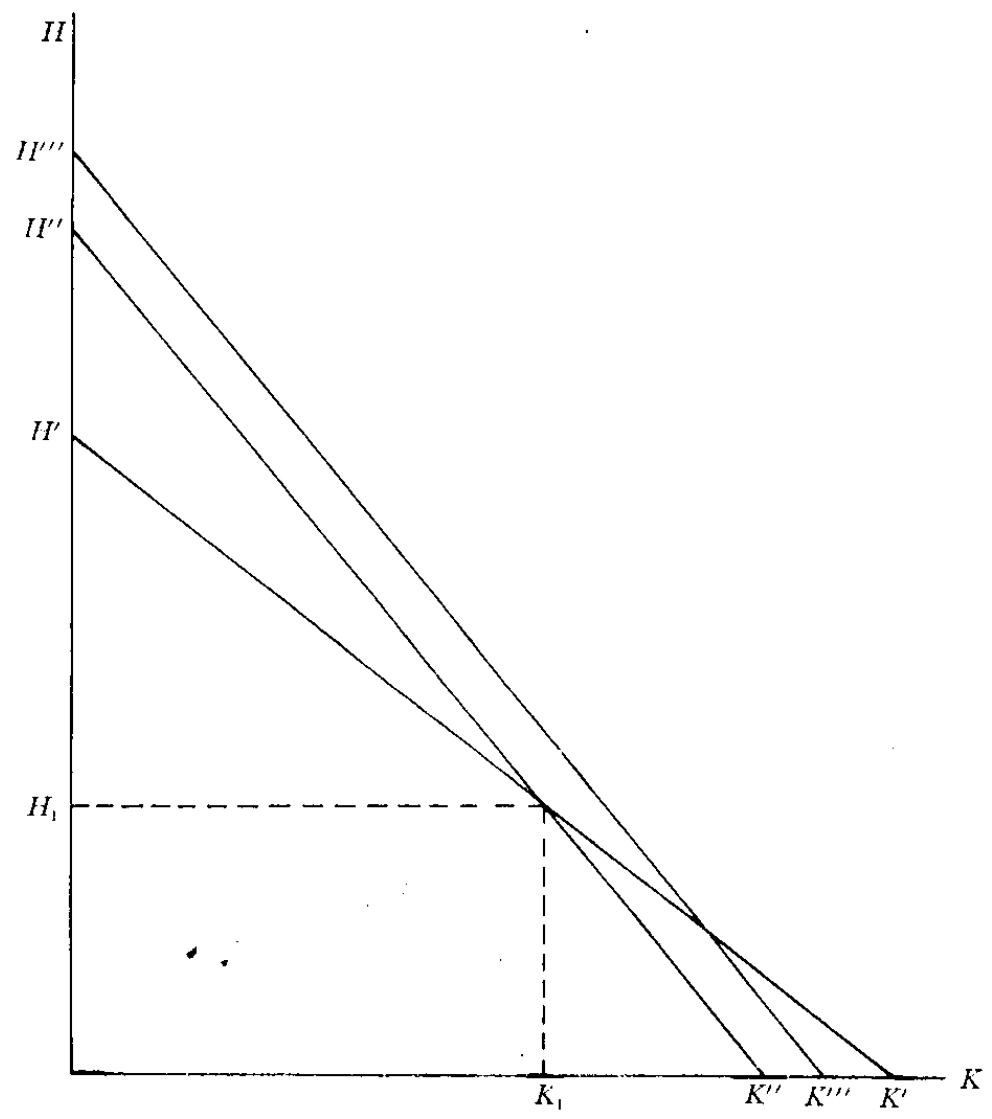


derivative.

The fifth term of the derivative, $\frac{\partial D}{\partial \theta}$, which has been assumed zero, can be further explored. The shift of funds from checking accounts to NOW accounts means the funds transferred are subject to lower reserve requirements because NOW accounts are regarded as savings accounts. If no change in hoarding and velocity is assumed, it will increase the wealth level and shift the budget line further out.

\section{CONCLUSION}

The analysis has shown the expected impacts of NOW accounts on the real investment mix the comparative static approach of a financial sector general equilibrium model. The results of this analysis confirmed the expectation that the adoption of NOW accounts will increase the amount of mortgage funds available and the level of housing, and will decrease the availability of other capital loans and the level of real capital except houses in economy.

The most important assumption made to obtain these results, in addition to the behavioral assumptions of each sector, is the adjacency property which says that the assets which are more closely related to the rates in question will be affected more when the rates in question change. An extreme case of this principle is the case of specialization considered earlier.

The wealth implication of NOW accounts was also considered to evaluate the desirability of its adoption for the economic objective of wealth maximization of the economy. The change in wealth from the introduction of NOW accounts, when wealth is defined as the present value of income streams from holding assets plus demand deposits, turned out to be positive. Relaxation of the constant level of deposit assumption will further increase the level of wealth in the economy.

The necessary condition for NOW accounts to have the effects described above is that there are the impediments in the supply side of the mortgage 
loan so that the introduction of NOW accounts will cause the transfer of funds from commercial banks to thrift institutions. The magnitude of the impact of NOW accounts on the real investment mix is also dependent on the way commercial banks get around the problem of eroded competitiveness in deposit acquisition. The issuance of NOW accounts by commercial banks to minimize the conversion and outflows of their deposits will reduce the overall impact of NOW accounts on the real investment mix. Even though this analysis cannot provide the magnitude or the speed of adjustment process, it verified that the adoption of NOW accounts nationally will produce a preferred real investment mix as well as an increased level of wealth.

Finally, it should be pointed out that this model can be utilized to evaluate any structural changes in financial institutions. Once we know how those structural changes will affect the demand or supply function of sectors involved, this model can tell us the steady-state solution for the movements of the interest rates and the associated levels of real capital investment.

\section{APPENDIX}

1. Several studies have been conducted by the Commission on Money and Credit(1961), the Hunt Commission(1971), and the Financial Institutions and the Nation's Economy (FINE) study (1976). These studies basically recommended that most of the operational powers of any depository intermediary be available to all of them, provided that the expanded powers operates under the same set of rules, which means the removal of regulations on asset and liability structure and on interest rates.

2. The elements in the matrix $A$ have the following sign pattern.

$$
\left(\begin{array}{llll}
- & + & + & + \\
+ & - & + & + \\
+ & + & - & + \\
+ & + & + & -
\end{array}\right)
$$

Since the diagonal elements are the "own partials" and the off-diagonal elements are "cross partials", the above sign pattern is obtainable directly from the behavioral assump- 
tion of gross substitutability. Each diagonal element is bigger, in absolute value, than the sum of the off-diagonal elements in the same column.

3. First, $A \cdot X=S$ was changed as follows:

$$
\begin{aligned}
& \left(\begin{array}{llll}
\frac{\partial M_{e}-\partial M_{f}}{\partial i_{m}} & \frac{\partial M_{e}-\partial M_{f}}{\partial i_{b}} & \frac{\partial M_{e}}{\partial r_{h}} & \frac{\partial M_{e}}{\partial r_{k}} \\
\frac{\partial B_{e}-\partial B_{f}}{\partial i_{m}}+\frac{\partial M_{e}-\partial M_{f}}{\partial i_{m}} & \frac{\partial B_{e}-\partial B_{f}}{\partial i_{b}}+\frac{\partial M_{e}-\partial M_{f}}{\partial i_{b}} & \frac{\partial B_{e}}{\partial r_{h}}+\frac{\partial M_{e}}{\partial r_{h}} & \frac{\partial B_{e}}{\partial r_{k}}+\frac{\partial M_{e}}{\partial r_{k}} \\
\frac{-\partial H_{e}}{\partial i_{m}} & \frac{-\partial H_{e}}{\partial i_{b}} & \frac{\partial\left(H-H_{e}\right)}{\partial r_{h}} & \frac{-\partial H_{e}}{\partial r_{k}} \\
\frac{-\partial K_{e}}{\partial i_{m}} & \frac{-\partial K_{e}}{\partial i_{b}} & \frac{-\partial K_{e}}{\partial r_{h}} & \frac{\partial\left(K-K_{e}\right)}{\partial r_{k}}
\end{array}\right) \\
& \left(\begin{array}{c}
\frac{d i_{m}}{d \theta} \\
\frac{d i_{b}}{d \theta} \\
\frac{d r_{k}}{d \theta} \\
\frac{d r_{k}}{d \theta}
\end{array}\right)=\left(\begin{array}{c}
\frac{\partial M_{f}}{d \theta} \\
0 \\
0 \\
0
\end{array}\right)
\end{aligned}
$$

By using the conditions of the balance sheet identity, the second row of the matrix $A$ can be written as follows.

$$
\begin{aligned}
& \frac{\partial B_{e}-\partial B_{f}}{\partial i_{m}}+\frac{\partial M_{e}-\partial M_{f}}{\partial i_{m}}=\frac{\partial D_{e}}{\partial i_{m}}+\frac{\partial H_{e}}{\partial i_{m}}+\frac{\partial K_{e}}{\partial i_{m}}-\frac{\partial D_{f}}{\partial i_{m}}-\frac{\partial H\left(r_{h}\right)}{\partial i_{m}}-\frac{\partial K\left(r_{k}\right)}{\partial i_{m}} \\
& \frac{\partial B_{e}-\partial B_{f}}{\partial i_{b}}+\frac{\partial M_{e}-\partial M_{f}}{\partial i_{b}}=\frac{\partial D_{e}}{\partial i_{b}}+\frac{\partial H_{e}}{\partial i_{b}}+\frac{\partial K_{e}}{\partial i_{b}}-\frac{\partial D_{f}}{\partial i_{b}}-\frac{\partial H\left(r_{h}\right)}{\partial i_{b}}-\frac{\partial K\left(r_{k}\right)}{\partial i_{b}} \\
& \frac{\partial B_{e}+\partial M_{e}}{\partial r_{k}}=\frac{\partial D_{e}}{\partial r_{h}}+-\frac{\partial H_{e}}{\partial r_{h}}+\frac{\partial K_{e}}{\partial r_{h}}-\frac{\partial H}{\partial r_{h}}-\frac{\partial K\left(r_{k}\right)}{\partial r_{k}} \\
& \frac{\partial B_{e}+\partial M_{e}}{\partial r_{k}}=\frac{\partial D_{e}}{\partial r_{k}}+\frac{\partial H_{e}}{\partial r_{k}}+\frac{\partial K_{e}}{\partial r_{k}}-\frac{\partial H\left(r_{h}\right)}{\partial r_{k}}-\frac{\partial K\left(r_{k}\right)}{\partial r_{k}}
\end{aligned}
$$

Through substitution and elimination process, $A \cdot X=S$ was simplified as shown.

4. Two simplifying assumptions of specialization and dichotomy show the relationship between the interest rates and their associated assets. Specialization means a close association between the mortgage rate and the demand for houses, and between the bond rate and the demand for other real capital. The extreme form of this association may be written as follows:

$$
\frac{\partial H_{e}}{\partial i_{b}}=0 \text { and } \frac{\partial K_{e}}{\partial i_{m}}=0
$$

Dichotomy assumes that there is no substitution between houses and other real capital in the portfolio of non-financial sector. This assumption is much stronger than that of spe- 
cialization and implies in the limit:

$$
\frac{\partial K_{e}}{\partial r_{h}}=0 \text { and } \frac{\partial H_{e}}{\partial r_{k}}=0
$$

Utilization of the above inequalities can simplify the expression of $\left|A_{3}\right|$ and $\left|A_{4}\right|$ a lot. It can be easily shown that the signs of $\left|A_{3}\right|$ and $\left|A_{4}\right|$ are negative and positive respectively, if we use either or both of the assumptions of specialization and dichotomy.

\section{REFERENCES}

Brainard, W., "Financial Intermediaries and a Theory of Monetary Control," Yale Economic Essay, 1964.

Feige, E. and Parkin, M., "The Optimal Quantity of Money, Bonds, Commodity Inventories and Capital," American Economic Review, June 1971.

Gurley, J. and Shaw, E., Money in a Theory of Finarce, The Ercckings Institutions, 1960.

Metzler, L., "Stability of Multiple Markets: The Hicks Condition," Econometrica, 13, October 1945.

Modigliani, F., Rasche, R. and Cooper, J., "Central Bank Policy, the Money Supply, and the Short-Term Rate of Interest," Journal of Money, Credit and Banking, May 1970.

Rao, D. and Kaminow, I., "Selective Credit Control and the Real Investment Mix: A General Equilibrium Approach," Journal of Finance, March 1974.

Santomero, A., "A Model of the Demand for Money by Households," Journal of Finance, March 1974.

Tobin, J., "A General Equilibrium Approach to Monetary Theory," Journal of Money, Credit and Banking, February 1969.

Tobin, J. and Brainard, W., "Financial Intermediaries and the Effectiveness of Monetary Controls," American Economic Review, May 1963. 